\title{
Experiências e tendências internacionais de modelos de cuidado para com o idoso
}

\author{
International Experiences and Trends \\ in Health Care Models for the Elderly
}

${ }^{1}$ Universidade Aberta da Terceira Idade, Universidade do Estado do Rio de Janeiro (UERJ). Rua São Francisco Xavier 524/ 10, Maracanã. 20559-900 Rio de Janeiro RJ. veras@uerj.br

\begin{abstract}
The profound socio-economic transformation occurring in Brazil is also driven by the demographic changes. Health expenditures will increase due to the aging population and increased use of health services. The National Agency for Supplementary Health published a resolution that encourages the participation of beneficiaries in health insurance programs for active aging, with the possibility of discounts in fees. Thus, the elderly now receive cash benefits to adhere to preventive health programs and early detection of diseases. We assessed the programs in the U.S. and UK, analyzing their successes and failings, which may assist in the formulation of health policies that are more appropriate and more cost-effective for the Brazilian elderly. This Opinion article seeks to demonstrate to Brazilian operators that it is time to change and to elicit discussions about the need for new strategies. A creative focus should be directed to the care of the elderly, who are the ones who suffer the effects of their own weakness and demand more health services.
\end{abstract}

Key words Aging, Health expenditures, Healthcare models, Health promotion, Maintenance of functional capacity
Resumo A profunda transformação socioeconômica do Brasilé impulsionada, inclusive, pela mudança demográfica. Gastos em saúde aumentarão devido ao aumento da população idosa e ao maior uso dos serviços de saúde. A Agência Nacional de Saúde Suplementar publicou uma resolução que incentiva a participação de beneficiários de planos de saúde em programas de envelhecimento ativo, com a possibilidade de descontos nas mensalidades. Assim, idosos passam a receber benefícios pecuniários para aderir a programas de saúde preventiva e de detecção precoce de doenças. Avaliamos as perspectivas nos Estados Unidos e no Reino Unido, analisando seus erros e acertos, aprendizado que talvez permita a formulação de ações de saúde mais adequadas para nossos idosos e com melhor relação custo-benefício. Este artigo de Opinião visa demonstrar às operadoras brasileiras que é hora de mudar e estimular a discussão sobre a necessidade de novas estratégias. O foco criativo deve ser dirigido ao cuidado dos idosos, que são os que mais sofrem os efeitos de sua própria fragilidade e os que mais demandam serviços de saúde.

Palavras-chave Envelhecimento, Gastos em saúde, Modelos assistenciais, Promoção da saúde, Manutenção da capacidade funcional 


\section{Introdução}

O envelhecimento populacional nunca esteve entre as principais preocupações em um país que é visto em todo o mundo como sinônimo de juventude. Mas isso vem mudando de forma drástica nas últimas décadas: o Brasil embarcou em um processo de desenvolvimento que o está levando a atingir índices sociais e demográficos de Primeiro Mundo, embora com sistemas e instituições herdadas de outro contexto.

O debate sobre essa transição tem sido parco até hoje, especialmente quanto a visões mais amplas, que buscam oportunidades e desafios em diversos setores. Assim, este artigo busca reduzir essa lacuna e incentivar a discussão sobre o envelhecimento populacional e suas conseqüências nas políticas sociais, particularmente na área da saúde, através de experiências internacionais, campo relativamente novo para o Brasil.

As rápidas mudanças demográficas em curso no Brasil representam, antes de tudo, uma enorme oportunidade para impulsionar seu crescimento e seu desenvolvimento social e econômico $^{1}$. O Brasil passa por um curto período, o chamado "bônus demográfico", único na história de cada nação, quando a força de trabalho é muito maior do que a população dependente ${ }^{2}$. Este é um ponto de inflexão que no Brasil deve durar apenas até 2020, mas cujos impactos durarão indefinidamente. Esse período é caracterizado por uma menor razão de dependência (relação entre o número de dependentes e pessoas em idade ativa). A razão de dependência, que tem declinado desde 1965, atingirá seu valor mínimo em 2020 e então começará a subir ${ }^{3}$. Em breve, iremos ingressar em um contexto demográfico fundamentalmente diferente, no qual a população cada vez mais madura colocará um peso extra sobre o sistema.

A velocidade do envelhecimento populacional no Brasil será significativamente maior do que ocorreu nas sociedades mais desenvolvidas no século passado. Por exemplo, foi necessário mais de um século para que a França visse sua população idosa aumentar de $7 \%$ para $14 \%$ do total. Em contraste, essa mesma variação demográfica ocorrerá nas próximas duas décadas (entre 2011 e 2031) no Brasil. A população idosa irá mais do que triplicar nas próximas quatro décadas, de menos de 20 milhões em 2010 para aproximadamente 65 milhões em 2050. Essa variação na estrutura etária da população resultará em maiores pressões fiscais sobre os sistemas públicos de saúde e previdência, enquanto a pressão fiscal sobre o sistema educacional financiado pelo governo diminuirá.

É provável que os gastos em saúde aumentarão substancialmente. De fato, cuidados com saúde tendem a emergir como um dos maiores desafios fiscais nas próximas décadas no Brasil ${ }^{4}$. Existem duas forças por trás da projeção de aumento dos gastos com saúde: o aumento da proporção e dos anos de vida de idosos na população e o aumento da intensidade do uso dos serviços de saúde pelos idosos ${ }^{5}$.

Espera-se que três fatores também aumentem o número de idosos necessitados de cuidados de longo prazo. Primeiro, o forte crescimento do número de pessoas muito idosas, nos próximos 30 anos, resultará em maior número absoluto de idosos fragilizados, mesmo considerando a redução da proporção de idosos fragilizados devido a avanços na prevenção de doenças e melhores práticas assistenciais. Segundo, a mudança de status das mulheres e a mudança nos valores sociais e familiares continuarão afetando a disponibilidade de apoio familiar para esses idosos. Projeções para o Brasil estimam que o número de pessoas sendo cuidadas por não familiares (cuidadores formais) irá duplicar até 2020, e será cinco vezes maior em 2040, em comparação com 2008. Em terceiro lugar, alguns fatores de risco que alcançavam majoritariamente o homem, principalmente o consumo do álcool e tabaco, e do estresse no trabalho, passarão também a atingir as mulheres, devido ao maior protagonismo do gênero feminino.

A organização do sistema de saúde no Brasil precisa ser ajustada para os diferentes perfis demográficos e epidemiológicos decorrentes do aumento da população idosa. A magnitude do aumento dos gastos em saúde com a população idosa dependerá essencialmente se esses anos a mais serão saudáveis ou de enfermidades e dependência. A prevenção e o retardamento de doenças e fragilidades, a manutenção da saúde, a independência e a autonomia em uma população mais velha serão os maiores desafios relacionados à saúde decorrentes do envelhecimento da população. Assim, qualquer política social e de saúde destinada aos idosos deve levar em conta a promoção da saúde e a manutenção da capacidade funcional.

Se, por um lado, os idosos apresentam maior carga de doenças e incapacidades, e usam mais os serviços de saúde, por outro, os modelos vigentes de atenção à saúde do idoso se mostram ineficientes e de alto custo, reclamando estruturas criativas e inovadoras. É por este motivo que, 
do ponto de vista da saúde pública, a capacidade funcional surge como o conceito de saúde mais adequado para instrumentalizar e operacionalizar uma política contemporânea de atenção à saúde do idoso. Essa política deve, portanto, ter como objetivo maior a manutenção da máxima capacidade funcional do indivíduo que envelhece, pelo maior tempo possível.

No Brasil, temos um fato novo em consonância com esta perspectiva de preservar a saúde e propiciar mais anos de vida saudável. A Agência Nacional de Saúde Suplementar (ANS) ${ }^{6}$ publicou, a Resolução Normativa no 265 , que incentiva a participação de usuários de planos de saúde em programas de envelhecimento ativo, com a possibilidade de descontos nas mensalidades. Ou seja, estimula-se a adesão dos beneficiários, além de incentivar as operadoras a adotarem essa perspectiva de cuidados. Assim, idosos passam a receber benefícios pecuniários para aderir a ações de saúde preventiva e detecção precoce de doenças. O objetivo da resolução é incitar o setor a se pautar pela prevenção ao invés do foco exclusivo no tratamento das doenças. A iniciativa é bem-vinda.

Como este fato é algo novo no país e em outros países existem algumas experiências neste campo, resolvemos fazer um apanhado do que está sendo realizado em dois países que possuem uma medicina de bom padrão - Estados Unidos e Reino Unido - para podermos avaliar seus erros e acertos, a fim de melhor conhecer essas vivências internacionais. Esse aprendizado talvez permita a formulação de ações de saúde mais adequadas para nossos idosos e com melhor relação custo-benefício.

\section{Os custos com cuidados de saúde nos Estados Unidos}

À medida que os formuladores de políticas de saúde consideram várias formas de atenção visando a conter os crescentes custos dos cuidados com saúde, é útil examinar os padrões de gastos em cuidados com saúde nos Estados Unidos.
É sabido que uma pequena proporção do total da população é responsável por metade de todos os gastos médicos nos EUA. Metade da população gasta pouco ou nada com saúde, enquanto $5 \%$ da população gastam quase a metade do total ${ }^{7}$. Em 2002, os 5\% da população norte-americana que gastaram mais com saúde representaram $49 \%$ dos gastos com saúde nos EUA. Destes, as despesas médicas anuais foram iguais ou superiores a US\$ 11.487 por pessoa. Em contraste, os $50 \%$ da população com as menores despesas representaram apenas 3\% do total de gastos médicos nos EUA com gastos médicos anuais abaixo de US\$ 664 por pessoa. Assim, aqueles incluídos nos 5\% gastaram, em média, mais de 17 vezes a mais por pessoa do que as pessoas na linha de base de 50\% (Tabela 1 ).

Em 2004, os EUA gastaram 16\% do seu produto interno bruto (PIB), em cuidados com saúde, cerca de US\$ 6.280 para cada americano. No entanto, o gasto total está distribuído de forma desigual nos diferentes segmentos da população, nas doenças específicas e por contribuintes. As despesas de saúde nos Estados Unidos subiram de US\$ 1.106 por pessoa em 1980, para US\$ 6.280 por pessoa em $2004^{10}$. Durante esse período, os custos dos cuidados com saúde cresceram mais rápido do que a economia como um todo. Como consequência, os gastos com saúde agora são responsáveis por $16 \%$ do PIB americano, em comparação a 9\% em 1980. Com o envelhecimento da população e o ritmo acelerado das inovações médicas, é provável que continue esta tendência.

Análises mais detalhadas dos padrões de gastos mudam com o tempo e afetam de maneira diferente os diversos tipos de contribuintes, tais como aqueles filiados ao Medicare, Medicaid, seguradoras privadas, os empregadores e consumidores. A pergunta que todos fazem é: como melhor empregar os esforços para conter os custos com saúde que não param de se elevar, e muito rapidamente ${ }^{10}$

Os que trabalham para desenvolver estratégias para reduzir ou conter custos consideram que os esforços devem ser direcionados para

\begin{tabular}{lrrrrrr}
\hline Tabela 1. Distribuição da população dos Estados Unidos por grupo de gastos entre $1977-2002$ \\
\hline \% população americana segundo gastos de saúde & $\mathbf{1 9 7 7}$ & $\mathbf{1 9 8 0}$ & $\mathbf{1 9 8 7}$ & $\mathbf{1 9 9 6}$ & $\mathbf{2 0 0 2}$ \\
\hline 1\% no topo & $27 \%$ & $29 \%$ & $28 \%$ & $28 \%$ & $22 \%$ \\
$5 \%$ no topo & $55 \%$ & $55 \%$ & $56 \%$ & $56 \%$ & $49 \%$ \\
$50 \%$ na base & $3 \%$ & $4 \%$ & $3 \%$ & $3 \%$ & $3 \%$ \\
\hline
\end{tabular}

Fontes: Berk \& Monheit ${ }^{8}$ e Yu \& Ezzati-Rice ${ }^{9}$ 
todo o sistema de saúde, com foco em áreas específicas e em aspectos do atendimento. Deste modo, alguns tópicos devem ser priorizados. Por exemplo, o aumento contínuo das despesas de saúde se deve ao aumento do custo do tratamento. O crescimento e envelhecimento da população têm papel significativo, com o aumento no número de pessoas tratadas pelas doenças mais caras e de longa duração. Essas informações lançam luz sobre áreas onde as mudanças na política podem trazer maior impacto.

Idosos (65 anos ou mais) eram 13\% da população dos EUA em 2002, mas consumiram 36\% do total do país em despesas em cuidados pessoais de saúde, segundo Keehan et al. ${ }^{11}$. A principal razão pela qual os cuidados com saúde estão espalhados mais uniformemente entre os idosos é que uma proporção muito maior de idosos do que os não idosos tem doenças crônicas onerosas.

É possível concluir que há um nivelamento substancial de despesas em uma população ao longo de vários anos, em comparação com apenas um único ano. Um episódio de pneumonia aguda ou um acidente de veículo a motor pode levar a uma hospitalização cara, mesmo numa pessoa normalmente saudável. Do mesmo modo, muitas pessoas têm doenças crônicas, tais como diabetes e asma, com tratamento bastante caro continuamente, mas que na maioria dos anos não os coloca no topo dos clientes em gastos de saúde. No entanto, a cada ano algumas das pessoas com doenças crônicas terão episódios ou complicações agudas que exigirão hospitalização ou outros tratamentos mais caros.

Os custos desembolsados podem impor um fardo financeiro significativo sobre os indivíduos e suas famílias. Essas despesas incluem franquias, copagamentos e pagamentos por serviços que não são cobertos por seguros de saúde. Nos Estados Unidos, as pessoas veem sua própria saúde fortemente associada com seu nível de despesas com ela. Analisando a população abaixo de 65 anos que tem empregador subsidiando o seguro de saúde, os pesquisadores não encontraram nenhuma diferença estatisticamente significativa na concentração entre os inscritos em planos de saúde apenas, e aqueles com planos com programa de gerenciamento e prestadores preferenciais ${ }^{12}$.

Algumas doenças têm tratamento muito oneroso. Em 2002, as cinco doenças mais caras eram: doenças cardíacas, câncer, traumas, distúrbios mentais e doenças pulmonares (Gráfico 1). As doenças cardíacas e traumas estão em primeiro e segundo lugar, respectivamente, como as duas

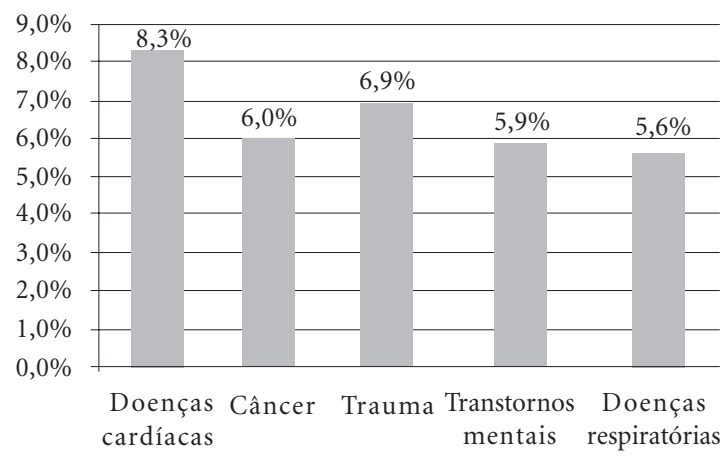

Gráfico 1. Percentual do total dos custos de saúde com as cinco condições (doenças) mais caras nos Estados Unidos em 2002

Fonte: Olin \& Rhoades ${ }^{12}$

situações mais caras em termos de despesa total de saúde. No entanto, com relação aos custos por pessoa, o câncer é a mais cara, ocupando as cardiovasculares o segundo lugar ${ }^{13}$. Juntas, essas cinco doenças foram responsáveis por uma proporção substancial dos gastos com saúde total em 2002. As 15 doenças mais caras nos Estados Unidos representaram $44 \%$ do total das despesas de saúde dos EUA em 1996.

Verificou-se que $25 \%$ da população dos EUA tinham uma ou mais das cinco principais doenças crônicas: transtornos do humor, diabetes, doença cardíaca, asma e hipertensão. O gasto para tratar essas cinco doenças ascendeu a US\$ 62,3 bilhões em $1996^{14}$. Além disso, pessoas com doenças crônicas tendem a ter outras fragilidades e doenças.

Um estudo mostrou que um pequeno número de doenças foi responsável pela maior parte do crescimento do gasto no total de cuidados com saúde entre 1987 e 2000, sendo que as cinco primeiras doenças (doenças cardíacas, doenças pulmonares, transtornos mentais, câncer e trauma) correspondem a $31 \%{ }^{15}$. O aumento do número de pessoas em tratamento de doenças caras teve impacto sobre o crescimento dos gastos de seguros privados semelhante aos custos de saúde em geral. O aumento do número de pessoas tratadas, ao invés da elevação dos gastos por caso tratado, foi o determinante mais importante do crescimento dos gastos de seguros privados, entre 1987 e 2002, de acordo com outro estudo ${ }^{16}$. 
Para 16 entre as 20 doenças mais caras, o aumento do número de pessoas sendo tratadas representou mais da metade do crescimento de despesas de saúde em seguros privados. Os pesquisadores atribuem o número adicional de pessoas que estão sendo tratadas a três fatores: (1) aumento contínuo de segurados adultos classificados como obesos; (2) mudanças nas orientações de tratamento e padrões para o tratamento de pacientes sem sintomas ou com sintomas leves apenas; e (3) a disponibilidade de novas tecnologias médicas para diagnosticar e tratar os pacientes.

Especialmente importante é o aumento no número de pessoas tratadas por problemas clínicos relacionados com a obesidade. De 1987 a 2002, a proporção da população atendida aumentou em $64 \%$ para a diabetes (representando $80 \%$ do aumento dos custos) e $500 \%$ para hiperlipidemia (representando quase 90\% do aumento dos custos). Muitos fatores podem explicar o aumento substancial nas taxas de tratamento para problemas ligados à obesidade: aumento no número de pessoas com doenças relacionadas à obesidade, aumento do número de pacientes mais gravemente doentes, ênfase maior em cuidados preventivos e introdução de opções de tratamento mais amplas.

As análises dos padrões de despesas com saúde nos Estados Unidos procuram esclarecer a melhor forma de concentrar os esforços para ajudar a conter o aumento de custos com saúde. O reconhecimento de que um grupo relativamente pequeno de indivíduos é responsável por uma grande fração dos gastos em Medicare, planos privados e a população como um todo deveria servir para desenhar modelos focados nesses grupos de risco. As pesquisas também continuam a aumentar a consciência da importância de doenças crônicas na despesa global como importante fator de aumento de custos, levando a programas de gerenciamento de doenças e outros esforços, tanto para melhorar a qualidade, quanto para reduzir os custos de enfermidades como diabetes, asma, hipertensão, doença cardíaca e obesidade.

Estudos apontam para diferenças importantes nas despesas com cuidados em saúde por área geográfica. Um estudo que dividiu o país em 306 regiões para tratamento de saúde descobriu que os pacientes nas áreas de maiores gastos recebem $60 \%$ mais cuidados ${ }^{17}$. Os autores descobriram que essas variações foram devidas não a diferenças de preços, severidade da doença ou condição socioeconômica, mas sim à quantidade total de serviços médicos prestados e às propor- ções relativamente mais elevadas de médicos e especialistas em regiões de alto custo.

Possivelmente, o maior problema dos modelos assistenciais americanos é o foco exclusivo na doença. Mesmo quando oferece um programa diferenciado, a proposta é voltada prioritariamente para a redução dos custos de doenças específicas, esquecendo que muitos pacientes possuem múltiplas patologias crônicas. Os dados americanos mostram inclusive o fracasso de tais programas, pois são realizados numa lógica hospitalocêntrica.

\section{Uma experiência interessante no Reino Unido}

Um exemplo emblemático das tentativas que estão sendo colocadas em prática no mercado de saúde pode ser observado no Reino Unido. Lá, os clientes de uma das maiores seguradoras, a PruHealth, recebem benefícios financeiros para deixarem seus carros parados e caminharem, usando o pedômetro com monitor cardíaco. Também obtêm descontos na compra de frutas e legumes em uma rede de supermercados associada à empresa de saúde e recebem incentivo financeiro para a prática de exercícios físicos em academias. Em síntese: um estilo de vida mais saudável pode levar à redução no valor da apólice de saúde.

A experiência inglesa não chegou ainda ao Brasil - e não se sabe se teria efeito positivo ou aceitação da sociedade. De qualquer forma, merece reflexão a mensagem passada: os planos de saúde operam numa espécie de mutualismo, em que os menos saudáveis consomem mais serviços médicos e inflacionam os valores pagos por quem está com sua capacidade funcional preservada e em boa forma. Por muitos anos, tivemos dificuldade de aceitar estímulos financeiros para cuidar de nossa saúde.

A aplicação do aporte epidemiológico e a ênfase em prevenção fazem com que esta nova abordagem favoreça a redução dos custos assistenciais em paralelo à ampliação da qualidade de vida, ao conferir prioridade à tecnologia do conhecimento e não à tecnologia das máquinas e imagens. Isso implica em reorganizar os serviços de saúde.

O Programa Vitalidade (The PruHealth Vitality Program) é um produto do seguro saúde da PruHealth, projetado para a promoção da saúde dos associados, para que estes sejam mais saudáveis e para recompensar financeiramente aqueles que o fazem, quer usem o plano ou não. 
Segundo a empresa prestadora, a razão para a criação desse programa é simples: as pessoas que se cuidam devem ser recompensadas através de seu plano de seguro saúde. Se os associados permanecem saudáveis, fazem exercícios regularmente, comem bem, não bebem e não fumam, é mais provável que usem menos o plano de saúde.

Uma das recomendações para os associados PruHealth é a realização da triagem básica e avaliação de saúde na Clínica de Diagnóstico. Um fato interessante é que existem algumas empresas parceiras que fazem essas avaliações de saúde, como por exemplo, o Boots Health Check, um serviço básico de triagem que acontece em grandes filiais da Boots (a maior rede de farmácia e conveniência do Reino Unido) e visa a avaliar o estilo de vida dos associados, podendo identificar certos riscos para a saúde, como pressão alta ou colesterol. Também oferece aos associados aconselhamento e orientação sobre como melhorar e manter um estilo de vida saudável.

A mesma lógica existe para as avaliações do preparo físico, que podem ser feitas em academias parceiras da PruHealth e são, segundo a empresa, uma ótima maneira de lançar um programa de condicionamento físico para o associado. Na área da nutrição, também há um programa bastante inovador, pois é sabido que alimentar-se com base em uma dieta equilibrada é uma das maneiras mais importantes pela qual se pode cuidar da saúde.

Ser saudável, para a empresa, deveria ser sua própria recompensa, mas ela entende que a maioria dos clientes necessita de motivação para começar. Portanto, todos os que participam do Programa Vitalidade têm direito a benefícios, e quanto maior for seu desempenho, maiores podem ser os benefícios. Eles incluem descontos em bilhetes de cinema, viagens pelo trem Eurostar e hospedagem de luxo nos spas Champneys Health, bem como generosas recompensas por ocasião da renovação. A imagem que a empresa deseja passar é a de que o Programa Vitalidade beneficia todos os clientes de todas as formas, com mais saúde e a custos mais baixos.

Em suma, podemos dizer que a empresa de saúde PruHealth radicaliza a cartilha do lifestyle, ou seja, a proposta da empresa é apostar neste achado da epidemiologia e repassar bonificação para que seus associados sigam de forma disciplinada a cartilha da vida saudável. Sabe-se que a pessoa estressada, que não faz exercício físico, tem hábitos alimentares inadequados, bebe e fuma, apresenta alta prevalência de doenças crônicas, então se deve fazer de tudo para evitá-las.
Para tal, os associados são bonificados e todos ganham, pois seus clientes estão vivendo mais e melhor, e a saúde financeira da PruHealth vai muito bem.

\section{Conclusão: Proposta contemporânea e adequada à realidade brasileira}

A descrição dos modelos internacionais aqui apresentados visa também a demonstrar às operadoras médico-hospitalares brasileiras que é hora de mudar. Na atualidade, muitas delas não veem com bons olhos essa mudança, apesar de ser justamente no segmento etário dos idosos que se dão as maiores despesas decorrentes de complicações de saúde agravadas pelo avanço da idade. A mensagem é simples e única: as empresas terão de se adaptar às novas tecnologias da moderna gerontologia, não é cabível manter a lógica da doença. Ao todo, cerca de 37 milhões, ou 81,3\% dos consumidores de planos de assistência médica, poderão receber bonificação se participarem de programas de promoção do envelhecimento ativo ao longo do curso da vida. O incentivo, agora, também é para o consumidor.

Com a nova proposta, a ANS faz uma tentativa de induzir a lógica preventiva no setor de saúde suplementar, cujo modelo de financiamento ainda é orientado para a execução de procedimentos. Lança mecanismos de indução também para os consumidores, cuja adesão é fundamental para o sucesso das ações de prevenção. Assim, o consumidor poderá ganhar duas vezes: evitando doenças e obtendo descontos e prêmios.

Mesmo não constituindo nenhuma concepção inovadora, pois, como vimos, vem sendo praticada em outros países, a proposta tem mérito, primeiramente por assumir as particularidades do idoso e colocá-lo como protagonista no sistema de saúde. Em segundo lugar, pelo entendimento da ANS da necessidade de induzir o projeto, ao compreender que a relação entre prestadoras e clientes de mais idade sempre foi de grande desconfiança.

Os prestadores nunca foram entusiastas da prestação de serviços para os idosos e sua política sempre foi tentar reduzir o peso desse segmento em suas carteiras. Os clientes idosos, por seu lado, sempre reclamaram das dificuldades de atendimentos e das glosas a muitos dos procedimentos solicitados, o que dificultava a utilização do seu plano de saúde, considerado muito caro por eles. Para as prestadoras, o valor pago por este segmento etário é muito reduzido e não 
cobre adequadamente os riscos de uma população com alta probabilidade de adoecer e impactar seus custos.

Aliás, este é um fato único. Não existe nenhuma relação de comércio em que vendedor e clientes têm posições tão antagônicas e que tudo fazem para se livrarem um do outro. Se fosse possível, as prestadoras de saúde não aceitariam esses clientes; e os idosos, por outro lado, sonham com um plano que cobrisse suas necessidades. Com esse clima beligerante e de hostilidade, sempre foi difícil sugerir e implantar alguma ação preventiva e/ou de monitoramento, pois a desconfiança da clientela sempre foi grande. Era comuns idosos se perguntarem por que eles iriam se submeter a ações preventivas (pois o cliente não é obrigado a conhecer sua maior vulnerabilidade), se o objetivo seria apenas ampliar os lucros das operadoras. Da parte das empresas de saúde, o interesse único era montar programas para um grupo bem reduzido, apenas para se posicionarem como empresas modernas e que oferecem o que há de mais moderno no campo do cuidado para o idoso (na verdade, não acreditavam no benefício de tais ações).

Programas de gerenciamento de doença para idosos têm uma relação custo-benefício baixa, pois os idosos possuem inúmeras doenças, e tratar adequadamente uma doença faz apenas reduzir os índices de morbidade de tal patologia. Para o idoso, no entanto, o problema não é resolvido, pois sua fragilidade é mantida e ele continuará com todos os demais agravos. A maioria das doenças crônicas dos idosos tem seu principal fator de risco na própria idade. Como envelhecer é uma conquista e não queremos evitá-la, a estratégia a ser empregada deve se concentrar na postergação do evento através de práticas preventivas ${ }^{18}$.

$\mathrm{Na}$ análise das informações americanas, percebe-se que apesar da enorme preocupação em conter custos, a abordagem se dá única e exclusivamente no foco da doença. Não negamos a ne- cessidade de ampliar a consciência sobre a importância de doenças crônicas e o aumento da despesa global, mas centrar os esforços apenas na redução dos custos é uma abordagem que não leva em consideração os erros nos modelos de cuidado.

Já a proposta inglesa tem um ingrediente bastante ousado e interessante, pois prioriza a promoção da saúde, o cuidado e a manutenção da autonomia. Deste modo, todas as ações desenvolvidas no programa visam, em última análise, à preocupação com a preservação da saúde do indivíduo idoso, procurando oferecer qualidade nos anos adicionais que serão vividos. Mesmo não sendo um programa desenhado apenas para este segmento etário, é lá que os melhores resultados, do ponto de vista financeiro, são alcançados por ser este o segmento que mais consome com saúde e, portanto, são os maiores gastadores. Pode-se considerar o projeto um aprofundamento de práticas preventivas, balizado pelo afã de detectar precocemente os agravos de saúde que acometem seus clientes.

A força da preocupação aqui apresentada está, genericamente, na compreensão da heterogeneidade que caracteriza a população idosa, no alto custo dos procedimentos de saúde a ela destinados, na diversidade dos gastos em subgrupos dessa população e na quantidade insuficiente de especialistas em geriatria. Por outro lado, seu valor se encontra também na certeza de que, sem uma abordagem preventiva e integral que associe a reflexão epidemiológica e o planejamento sistemático de ações de saúde, não há saída possível para a crise de financiamento e reestruturação do setor.

Estas reflexões têm por objetivo estimular a discussão para a necessidade de novas estratégi$\mathrm{as}^{19}$. Um foco inovador e criativo deve ser dirigido ao cuidado do idoso e aos que têm doença crônica, que são os que mais sofrem os efeitos de sua própria fragilidade e os que mais demandam serviços de saúde. 


\section{Referências}

1. Lee R, Mason A. Fertility, human capital, and economic growth over the demographic transition. European journal of population 2010; 26(2):159-182.

2. Bloom DE, Canning D, Fink EG. Population aging and economic growth. In: Spence M, Leipziger D, editors. Global challenges and growth. Washington, DC: The World Bank; 2009.

3. Queiroz BL, Turra CM. Window of opportunity: socioeconomic consequences of demographic changes in Brazil. Washington, DC: NTA; 2010.

4. Banco Mundial. Population aging: is Latin America ready? Directions in development. Washington, DC: The World Bank; 2011.

5. National Institute of Aging. National Institutes of Health, US Dept. of Health \& Human Services. Why population aging matters: a global perspective. Washington DC: NIA; 2007.

6. Agencia Nacional de Saúde Suplementar (ANS) Resolução Normativa no 265 de 19 de agosto de 2011. Dispõe sobre a concessão de bonificação aos beneficiários de planos privados de assistência à saúde pela participação em programas para Promoção do Envelhecimento Ativo ao Longo do Curso da Vida e de premiação pela participação em programas para População-Alvo Específica e programas para Gerenciamento de Crônicos. Diário Oficial da União 2011; 22 ago.

7. Conwell LJ, Cohen JW. Characteristics of people with high medical expenses in the U.S. civilian noninstitutionalized population, 2002. Statistical brief 73. 2005 March Rockville, MD: Agency for Healthcare Research and Quality. [cited June 2011]. Available from: http://www.meps.ahrq.gov/PrintProducts/ PrintProdLookup.asp?ProductType $=$ StatisticalBrief.

8. Berk ML, Monheit AC. The concentration of health expenditures, revisited. Health Aff 2001; 20(2):9-18.

9. Yu WW, Ezzati-Rice TM. Concentration of health care expenses in the U.S. civilian noninstitutionalized population. Statistical Brief 81. 2005 May (cited 07 Sept 2011). Rockville, MD: Agency for Healthcare Research and Quality. Available from: http://www. meps.ahrq.gov/PrintProducts/PrintProdLookup. asp? ProductType $=$ StatisticalBrief.

10. Centers for Medicare \& Medicaid Services. Office of the Actuary. National health expenses aggregate and per capita amounts, percent distribution, and average annual percent growth, by source of funds: selected calendar years 1980-2003 [cited June 2011] Available from: http://www.cms.hhs.gov/NationalHealthExpendData/02_NationalHealthAccounts Historical.asp\#TopOfPage.
11. Keehan SP, Lazenby HC, Zezza MA, Catlin AC. Age estimates in the national health accounts. Health care financing review 2004; 1(1).

12. Cohen S, Yu W. The persistence in the level of health care expenditures over time: estimates for the U.S. population, 2002-2003. Statistical Brief 124 2006 May Rockville, MD: Agency for Healthcare Research and Quality. [cited June 2011]. Available from: http://www.meps.ahrq.gov/PrintProducts/ PrintProdLookup.asp? ProductType $=$ StasticalBrief.

13. Olin GL, Rhoades JA. The five most costly medical conditions, 1997 and 2002: estimates for the U.S. civilian noninstitutionalized population. Statistical Brief 80. 2006 Rockville, MD: Agency for Healthcare Research and Quality. [cited June 2011]. Available from: http://www.meps.ahrq.gov/PrintProducts/PrintProd Lookup.asp?ProductType $=$ StatisticalBrief.

14. Druss BG, Marcus SC, Olfson M, Tanielian T, Elinson L, Pincus HA. Comparing the national economic burden of five chronic conditions. Health Aff 2001; 20(6):233-241.

15. Thorpe KE, Florence CS, Howard DH, Joski P. The rising prevalence of treated disease: effects on private health insurance spending. Health Aff 2005; (Supl.):W5-317-W5-325.

16. Thorpe KE, Florence CS, Joski P. Which medical conditions account for the rise in health care spending? Health Aff 2004; (Supl.): W4-437-45.

17. Fisher ES, Wennberg DE, Stukel TA, Gottlieb DJ, Lucas FL, Pinder EL. The implications of regional variations in Medicare spending. Part 1: The content, quality, and accessibility of care. Ann Intern Med 2003; 138(4):273-287.

18. Veras RP, Parahyba MICA. O anacronismo dos modelos assistenciais para os idosos na área da saúde: desafios para o setor privado. Cad Saude Publica 2007; 23(10):2479-2489.

19. Azevedo ERFC. Os desafios das operadoras de planos de saúde de autogestão em um cenário de envelhecimento populacional e cronificação de doenças [monografia]. Rio de Janeiro (RJ): Rede de Ensino Luiz Flávio Gomes; 2010.

Artigo apresentado em 25/10/2009

Aprovado em 21/02/2010

Versão final apresentada em 15/03/2010 